\title{
A persuasive intervention: improving the compliance of extended venous thromboembolism prophylaxis following cancer resections in a tertiary colorectal and hepatobiliary unit
}

\author{
Yao Pey Yong, Alvin Karangizi, Ayan Banerjea \\ Queen's Medical Centre, England
}

\begin{abstract}
Extended venous thromboembolism (VTE) prophylaxis has been shown to reduce the incidence of VTE in patients following cancer resections.[1] However, ensuring patients are discharged with the prescription remains a challenge, with junior doctors frequently rotating throughout different specialties. We conducted an audit to assess the compliance rate in the colorectal and hepatobiliary (HPB) unit at the Queen's Medical Centre in Nottingham.

Extended VTE prophylaxis was considered compliant to the guideline if it was prescribed on discharge. The baseline measurement demonstrated compliance rates of $79 \%$ and $48 \%$ in the colorectal and HPB units respectively. Following discussion with the stakeholders, several interventions that include education and visual reminders were implemented to increase awareness of the importance of extended VTE prophylaxis among junior doctors. Results of the re-audit have shown a remarkable improvement; compliance rates were increased to $93 \%$ and $72 \%$ in the colorectal and HPB units respectively.
\end{abstract}

We conclude that visual reminder is a simple yet effective tool to improve awareness among junior doctors on the importance of extended VTE prophylaxis in cancer patients. Nevertheless, education remains crucial to ensure the sustainability of any intervention.

\section{Problem}

There has been an ongoing issue with extended VTE prophylaxis not being prescribed on the to-take-out (TTO) prescription in patients who have had cancer resections. This is due to the lack of awareness among the junior doctors as they frequently rotate through different specialties during their short placements. Cases have been identified by general practitioners as well as surgical nurse practitioners when patients receive telephone follow-up. This raises concern over patient safety as the lack of extended VTE prophylaxis inevitably increases the risk of VTE.

\section{Background}

VTE remains a significant burden to the NHS. It causes more inhospital mortality than the combined annual deaths from cancer and road traffic accidents.[2] Its main complications include pulmonary embolism and post-thrombotic syndromes, which include chronic oedema and ulceration of the lower limbs. The risks of VTE can be reduced by chemical and mechanical prophylaxis. Extended prophylaxis given post-operatively for 28 days following major abdominal and pelvic cancer surgery has been shown to significantly reduce the incidence of VTE.[1]

Queen's Medical Centre in Nottingham is a central teaching hospital and a tertiary centre in the East Midlands. It is a high volume centre with approximately 350 elective bowel resections performed each year. Both the colorectal and HPB unit have their own dedicated 28 bedded ward, in addition to a 24 bedded surgical high dependency unit and a 21 bedded intensive care unit. The extended VTE prophylaxis was implemented in the colorectal and hepatobiliary (HPB) unit since 2012. It is indicated in all patients who have undergone abdominal and pelvic cancer resections as recommended. The protocol to prescribe extended VTE prophylaxis is available on the trust's intranet. However, it is not widely publicised and the majority of junior doctors new on the rotation are not aware of this guideline. Prior to this audit, a verbal reminder was the sole method of ensuring compliance.

A variety of innovative methods have been proposed to improve the compliance of extended VTE prophylaxis. These include educating junior doctors to instill the importance of extended VTE prescription, putting up posters in doctors' offices as a reminder, and incorporating a reminder to the hospital IT system.[3,4,5]

\section{Baseline measurement}

An audit was performed to assess the compliance of extended VTE prophylaxis in patients who have undergone cancer resections in the colorectal and HPB units between July and September 2013. Patients were retrospectively selected at random from the cancer database. Data were collected from discharge summaries available on the hospital electronic system. These included patient demographics, type of operation, prescription, and duration of extended VTE prophylaxis.

A total of 25 patients were identified from each unit respectively. One or two patients were excluded from each unit as these patients 
were on long-term anti-coagulation and resumed their usual medication post-operatively. The extended VTE prophylaxis was considered compliant to the guideline if it was prescribed on the TTO on discharge. The compliance rate of extended VTE prophylaxis in the colorectal unit was 79\% (19/24) and 48\% (11/23) in the HPB unit.

See supplementary file: ds4090.png - "Chart1"

\section{Design}

Following presentation of the audit results at the audit meeting, several interventions were suggested by the stakeholders with input from the junior doctors. In addition to educating junior doctors to raise awareness, visual cues were felt to be equally important as a reminder.

The initial idea was to design a sticker to be placed on the drug card to highlight the need for extended VTE prophylaxis. Moreover, a poster that is to be placed in the doctors' office may further emphasise the importance of extended VTE prophylaxis to junior doctors.

All designs have been reviewed by the consultant surgeon responsible for clinical governance as well as the surgical nurse practitioners.

\section{Strategy}

PDSA cycle 1: A sticker to highlight extended VTE prophylaxis was proposed to be placed on the existing VTE prophylaxis section on the drug card. The purpose is to serve as a visual reminder for junior doctors. We approached the trust medicine management group on this issue and were informed that it required approval by the VTE group. However, the VTE group had no service meetings for the next six months. Given the urgency of tackling the issue, it was decided that we would focus on other methods to improve compliance whilst waiting for their meeting.

PDSA cycle 2: A poster was designed to be placed in the doctors office as a reminder to prescribe extended VTE prophylaxis. The initial draft was reviewed by consultant and nurse practitioners. The general consensus was that it might not be visible as there are already several posters for other clinical purposes on the wall. Therefore the poster was redesigned to a smaller size to fit next to the computer screen. These were printed in bright colours for visibility and laminated to increase durability. The costs of these mini posters were covered by the colorectal department.

PDSA cycle 3: The redesigned poster placed next to the computer screen has a higher visibility and is hence better at reminding junior doctors to include extended VTE prophylaxis when TTOs are being completed electronically. A reminder for routine electronic VTE assessment was also incorporated to the mini poster at the same time.

PDSA cycle 4: The results of the initial audit and recommendations were sent to all surgical junior doctors via email. In addition, a briefing on extended VTE prophylaxis will be incorporated at every departmental induction meeting for the new cohort of junior doctors.

\section{Results}

A further audit to close the loop was performed two months after the implementation to help improve compliance on extended VTE prophylaxis. The same methodology was used for the second audit.

A total of 15 patients were selected for each subspecialty. In the colorectal unit, the compliance rate was increased to $93 \%(14 / 15)$ and $72 \%(11 / 15)$ for the HPB unit. This has shown significant improvement compared to the initial audit.

See supplementary file: ds4089.jpg - "VTE"

\section{Lessons and limitations}

We did not have the opportunity to assess the feasibility of implementing sticker to highlight the need for extended VTE prophylaxis on the drug card. This was due to the infrequent meeting of the VTE group and the urgency of the issue. In addition this, we were also informed by the management that stickers are currently being used by anaesthetists to prescribed intra- and postoperative medications. Having more stickers on the drug card may reduce the visibility of those prescribed by anaesthetists. Due to this reason, we thought perhaps this method may be more useful in centers where stickers are not already being used routinely on the drug card. However, we still hope to discuss the possibility of placing stickers on the drug card with the VTE group and set up a reminder on the trust's IT system.

The idea of a poster was raised following discussion between stakeholders and junior doctors. Prior to committing to this intervention, we decided to inspect the doctors' office to assess its feasibility. We found that there were already a number of posters on the walls in the office and placing another one would not be efficient for its purpose. This might not have been noticed if we have not take the extra step to ensure the practicability of the proposed intervention. It was due to this reason that the extended VTE prophylaxis poster was redesigned to be placed next to the computer screen.

Improvements were made at each step to ensure extended VTE prophylaxis was prescribed and the interventions are sustainable. These included briefings at induction and a mini poster to act as a reminder during the completion of discharge summaries. We found that a visual reminder is a simple yet effective way of improving awareness among junior doctors on the importance of extended VTE prophylaxis in cancer patients. Nevertheless, education remains crucial to ensure the sustainability of any intervention.

\section{Conclusion}

We have identified an issue with the prescription of extended VTE prophylaxis in cancer patients on discharge in the initial audit and 


\section{BMJ Quality Improvement Reports}

have demonstrated that our interventions have achieved a significant improvement in patient care. These quality improvements have resulted in better care by ensuring postoperative cancer patients receive extended VTE prophylaxis to reduce the risk of VTE.

\section{References}

1. National Institute for Health and Clinical Excellence (NICE) (2010) Venous thromboembolism prevention quality standard.Available at: http://www.nice.org.uk/guidance/qs3/chapter/qualitystatement-7-extended-vte-prophylaxis [Accessed: 20 September 2014]

2. Fitzmaurice DA, Murray E. Thromboprophylaxis for adults in hospital. BMJ 2007;334(7602):1017-18

3. Patel M, Harris M, Tapply I, Longman R. Improving adherence to guidelines for extended venous thromboembolism prophylaxis in patients with colorectal cancer. BMJ Qual Improv Report 2013;2: doi:10.1136/bmjquality.u200573.w1062

4. Watts $L$ and Grant $D$. Venous thromboembolism (VTE) risk assessment and prophylaxis in acute orthopaedic admissions: improving compliance with national guidelines. BMJ Qual Improv Report 2013. 2: doi:10.1136/bmjquality.u202229.w1118

5. Sinha P, Najefi A, Hambidge J. A simple measure to improve the rates of thromboprophylaxis prescription post surgical fixation of neck of femur fractures in a district general hospital. BMJ Qual Improv Report 2014. 3: doi:10.1136/bmjquality.u202991.w1956

\section{Declaration of interests}

Nothing to declare.

\section{Acknowledgements}

Mr lan Beckingham and Queen's Medical Centre clinical audit department. 